\title{
Descentralización de los escenarios de enseñanza-aprendizaje de los posgrados de Ginecotocología. Experiencia de la Clínica Ginecotocológica A
}

\author{
Fernanda Nozar* ${ }^{\star}$, Leonel Briozzo ${ }^{\dagger}$, Verónica Gallino, Verónica Fiol*, Miriam Piastri§, \\ Francisco Coppola", Natalia Sica*, Víctor Rechi**, Eric Quevedo**
}

\section{Resumen}

La Clínica Ginecotocológica A (CGA) de la Facultad de Medicina (FM) se ha comprometido con los objetivos de la reforma sanitaria en lo referente a la formación de posgrados, trabajando para ello en la descentralización de los escenarios de enseñanza-aprendizaje. En esta publicación se presenta la estrategia de descentralización de la formación de posgrados de ginecotocología de la CGA en el período 2010-2018. Se describen las herramientas que permiten mantener el contacto de los posgrados y tutores sustentados en tecnologías de la comunicación para realizar regularmente ateneos, actividades tutoriales, teleclínicas. Por otro lado, se describe la distribución logrado de postgrados, así como el logro de las destrezas en los diferentes Centros Docentes Asociados (CEDA). De la evaluación del funcionamiento de la red de CEDA de la CGA, así como de los logros formativos de recursos humanos, se concluye que es factible el aumento del cupo formativo de posgrados de Ginecotocología apelando a la descentralización estructurada de los escenarios de aprendizaje.

Palabras clave: Educación de posgrado

Educación médica continua

Key words: $\quad$ Education, graduate

Education, medical, continuing

\footnotetext{
* Profesora Adjunta. Clínica Ginecotocológica A. Facultad de Medicina. Universidad de la República. † Profesor. Clínica Ginecotocológica A. Facultad de Medicina. Universidad de la República.

$\ddagger$ Asistente de clínica. Clínica Ginecotocológica A. Facultad de Medicina. Universidad de la República. $\S$ Jefe de Residentes. Clínica Ginecotocológica A. Facultad de Medicina. Universidad de la República. TI Profesor Agregado. Clínica Ginecotocológica A. Facultad de Medicina. Universidad de la República.

** Médico colaborador. Tutor. Clínica Ginecotocológica A. Facultad de Medicina. Universidad de la República.

Correspondencia: Dra. Fernanda Nozar. Bulevar Artigas 1550, Montevideo. Uruguay.

Correo electrónico: fernandanozar@gmail.com

Los autores declaran no tener conflicto de interés.

Recibido 7/1/2019

Aprobado 23/5/2019
} 


\section{Introducción}

En la misión de la Facultad de Medicina (FM) de la Universidad de la República (Udelar) se resaltan compromisos formativos, de investigación, de incorporación de la actividad en la comunidad y se resalta también la contribución al desarrollo de un sistema nacional de salud universal, equitativo y solidario ${ }^{(1)}$.

En tal sentido, el proceso de formación de recursos humanos por parte de la FM tiene necesariamente que ajustarse e incidir en el sistema sanitario vigente, así como en las necesidades del país.

La ley del Sistema Nacional Integrado de Salud (SNIS) del año 2007 ha generado un cambio en la estrategia de atención sanitaria en Uruguay, donde se prioriza la Atención Primaria en Salud y el Primer Nivel de Atención. En este escenario, el Ministerio de Salud actúa como órgano rector del sistema en todos sus aspectos, incluidos los recursos humanos ${ }^{(2,3)}$.

La Escuela de Graduados de la FM se proyecta en la misma dirección, es decir hacia el desarrollo y sostén de nuestro SNIS. Desde el inicio de la reforma sanitaria actual, ha favorecido la formación con orientación generalista sin límite de ingreso en la mayoría de las especialidades, manteniendo cupos limitados para aquellas que requieren la incorporación de destrezas específicas en las cuales es necesario asegurar este proceso formativo, como son las especialidades quirúrgicas y dentro de estas últimas podemos incluir a la Ginecotocología.

Por otro lado, debemos destacar la ley de residencias médicas vigente que especifica que se promoverá el proceso de descentralización de las unidades docentes responsables de la FM de la Udelar, con lo cual los servicios formadores de residentes deberán adecuar su funcionamiento para dar cumplimiento a la misma ${ }^{(4)}$.

En este contexto es que la Clínica Ginecotocológica A (CGA) de la FM se compromete con los objetivos en lo referente a la formación de posgrados, tendiendo a la formación integral de especialistas preparados para cubrir adecuadamente las necesidades del SNIS, pero además se compromete con la descentralización de los escenarios de enseñanza-aprendizaje, con el objetivo de lograr contar con especialistas integralmente formados a lo largo y ancho del país.

Por todos los puntos expuestos consideramos relevante la difusión de la estrategia y la experiencia de descentralización de la formación de posgrados de Ginecotocología desarrollada por la CGA.

\section{Descripción de la estrategia y experiencia de descentralización de la formación de posgrados en el período 2010-2018}

En este período se han acreditado por la Escuela de Graduados de la FM, e incorporado al funcionamiento de la

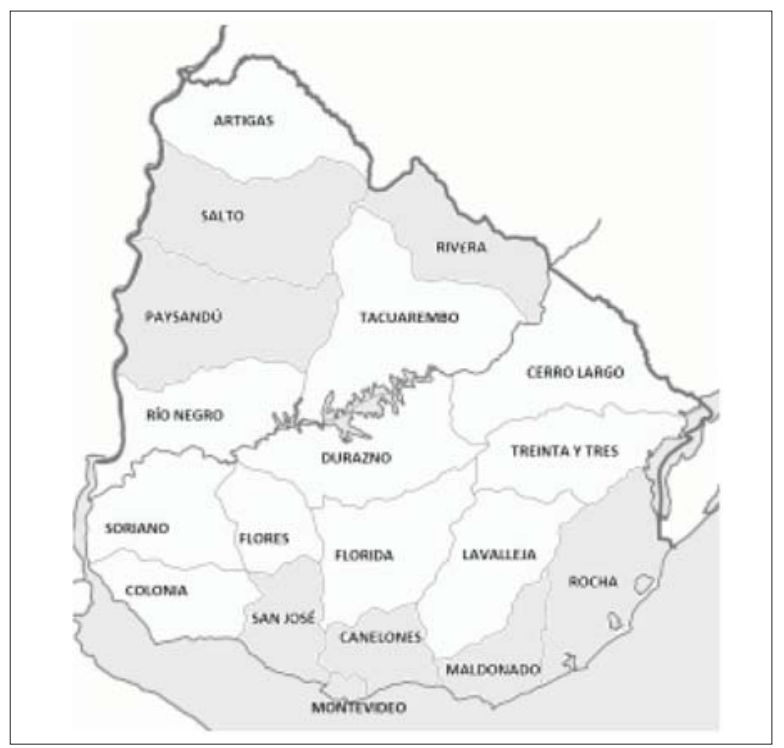

Figura 1. En sombreado se indican los departamentos del país donde se cursan posgrados de Ginecotocología de la Clínica Ginecotocológica A.

CGA, los siguientes Centros Docentes Asociados (CEDA): en Montevideo, Servicio Médico Integral (SMI); en el interior del país, Hospital Escuela del Litoral de Paysandú, Hospital Regional de Salto, Asociación Médica de San José, Hospital de Maldonado, Cooperativa Asistencial Médica de Rivera, Corporación Médica de Paysandú, Corporación Médica de Canelones, Colectivo Médico Rochense, el Hospital de Rivera y el Hospital Regional de Salto. Así se ha desarrollado una red de CEDA que permite efectivizar la descentralización formativa de especialistas. La distribución de todos los centros formadores de posgrados de la CGA se muestra en la figura 1.

Desde el año 2011, y en vista a lograr uniformidad en la formación de los posgrados en los diferentes escenarios de enseñanza-aprendizaje, se han desarrollado diversas herramientas que permiten de forma descentralizada mantener el contacto de los posgrados y tutores/docentes asociados con los integrantes de la CGA, apostando fundamentalmente a la comunicación regular de los diferentes equipos de trabajo. La base de esta uniformización en la formación del posgrado se sustenta en los lineamientos formativos planteados en el Libro de Postgrado de la Clínica desarrollado para tal fin ${ }^{(5)}$. En el mismo están claramente explicitados el perfil del ginecotocólogo, las áreas formativas, los objetivos de aprendizaje, así como la metodología.

Tanto para las actividades docentes tutoriales como para las actividades académicas y asistenciales que significan los ateneos, se han utilizados herramientas de comu- 
Tabla 1. Distribución evolutiva entre los años 2010-2018 de posgrados. Montevideo/interior.

\begin{tabular}{lccccccccc}
\hline & 2010 & 2011 & 2012 & 2013 & 2014 & 2015 & 2016 & 2017 & 2018 \\
\hline Montevideo: & 5 & 9 & 9 & 8 & 6 & 8 & 5 & 7 & 7 \\
CHPR & 4 & 6 & 7 & 6 & 4 & 7 & 4 & 5 & 6 \\
Otro & 1 & 3 & 2 & 2 & 2 & 1 & 1 & 2 & 1 \\
Interior: & 0 & 2 & 1 & 1 & 5 & 4 & 7 & 5 & 7 \\
Total & 5 & 11 & 10 & 9 & 11 & 12 & 12 & 12 & 14 \\
\hline
\end{tabular}

nicación periódicas a distancia. Esas herramientas corresponden a comunicaciones por la sala de telemedicina de la Fundación Peluffo Giguens, por comunicación a través del programa $\mathrm{ZOOM}^{\circledR}$, así como a través de teleclínicas en el marco del Proyecto ECHO (Extension of Community Health Outcomes), cuya premisa es democratizar el conocimiento y descentralizar la atención ${ }^{(6)}$.

Con frecuencia semanal se realizan ateneos clínicos con participación del staff docente de la CGA, los equipos profesionales de todos los CEDA y de todos los posgrados. En estos ateneos semanales se presentan y discuten casos clínicos desde los diferentes CEDA, permitiendo compartir conocimiento, realizar actualizaciones y articular aspectos asistenciales en función del nivel de complejidad de cada centro, apuntando a lograr la resolución de los problemas asistenciales a nivel local y regional cuando esto es posible, es decir, reafirmando la descentralización no solo en la formación de recursos humanos sino también a nivel asistencial.

Por otro lado, también con frecuencia semanal y con el programa $\mathrm{ZOOM}^{\mathbb{B}}$ se realizan actividades docentes de aprendizaje basadas en problemas ${ }^{(7)}$ con un tutor responsable siguiendo el programa de formación de posgrado $^{(5)}$. Estas actividades involucran obligatoriamente a todos los posgrados de la clínica.

En lo que tiene que ver con actividades académicas presenciales, regularmente durante el año en los diferentes CEDA, los docentes de la CGA participan junto con los referentes locales en jornadas de actualización y entrenamiento en temas relevantes para la asistencia y formación en dichos centros, apuntando tanto a la enseñanza de posgrados, a la formación médica continua y también, aprovechando estas instancias para el contacto con los responsables técnicos de las instituciones y representantes de la sociedad civil local, promover las actividades de extensión universitaria.

En la tabla 1 y la figura 2 se muestra la distribución de los posgrados de ginecología de la CGA en el período 2010-2018. En Montevideo, discriminados entre el Centro Hospitalario Pereira Rossell (CHPR) y otros

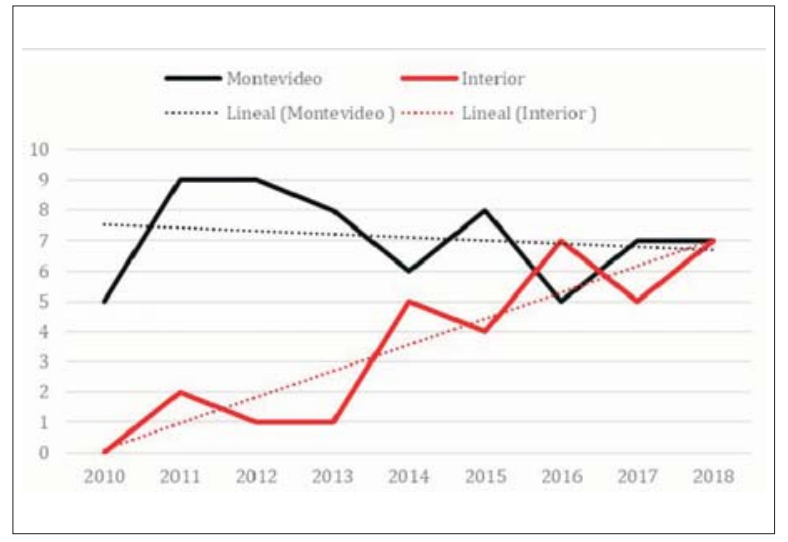

Figura 2. Distribución evolutiva entre los años 2010-2018 de posgrados. Montevideo/interior.

centros. Se destaca que hasta el año 2013 los otros centros correspondían al Hospital Militar y al Hospital Policial y desde el 2014 al SMI. En el interior del país se incluyen los CEDA de la Administración de los Servicios de Salud del Estado (ASSE) y de la Federación Médica del Interior (FEMI). Se puede visualizar la línea evolutiva, con una clara tendencia creciente de la formación en los CEDA del interior del país. Al momento actual, de la totalidad de posgrados que se encuentran cursando (se incluyen las generaciones 2016, 2017 y 2018), están distribuidos 50\% en Montevideo y 50\% en el interior de un total de 38 posgrados que se encuentran cursando diferentes semestres. Por lo tanto, en el presente podemos afirmar que la CGA pasó de tener prácticamente centralizada totalmente la formación de especialistas en el CHPR, a contar con más médicos cursando sus posgrados en otros CEDA (23 de 38 posgrados).

En una especialidad donde el aprendizaje y la adquisición de destrezas es imprescindible para su ejercicio, creemos relevante presentar el número de procedimientos vinculados a la especialidad realizados por los diferentes posgrados. Para esto, mostramos en la tabla 2 el 


\begin{tabular}{|c|c|c|c|c|c|}
\hline & CHPR & SMI & Paysandú & Maldonado & AMSJ \\
\hline PV & 272 & 334 & 178 & 303 & 172 \\
\hline $\mathrm{PI}$ & 19 & 19 & 7 & 24 & 4 \\
\hline CST & 178 & 235 & 226 & 180 & 58 \\
\hline LEC & 56 & 44 & 10 & 52 & 40 \\
\hline HTA & 10 & 22 & 32 & 10 & 5 \\
\hline HTV & 8 & 12 & 10 & 17 & 4 \\
\hline LP-U & 9 & 25 & 10 & 7 & 11 \\
\hline LP-C & 25 & 38 & 44 & 21 & 7 \\
\hline
\end{tabular}

PV: parto vaginal; PI: parto instrumental; CST: operación cesárea; LEC: legrado evacuador complementario; HTA: histerectomía abdominal; HTV: histerectomía vaginal; LP-U: laparoscopía de urgencia; LP-C: laparoscopía de coordinación.

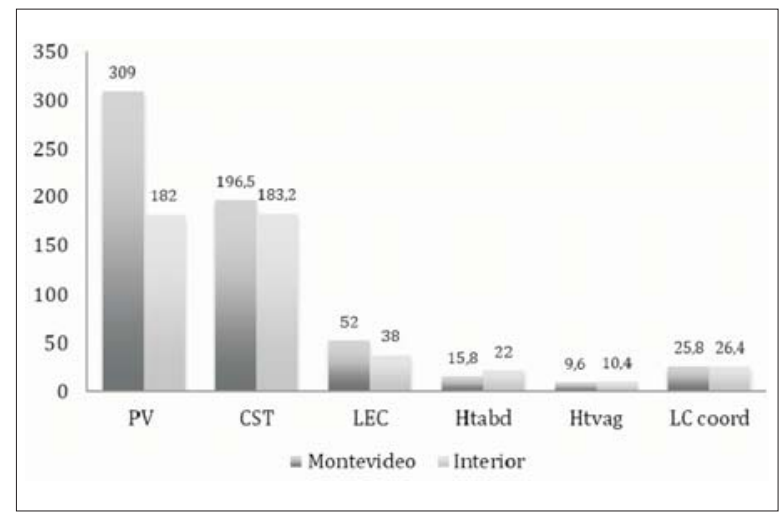

Figura 3. Promedio de procedimientos gineco-obstétricos Montevideo (gris oscuro) versus interior (gris claro).

promedio de procedimientos realizados por los 11 médicos que finalizaron su posgrado en el año 2017.

En la figura 3 se muestra el promedio de procedimientos realizados en los CEDA de Montevideo (CHPR y SMI) versus interior (CEDA de otros departamentos), y podemos ver que el promedio en algunos casos es similar, cuando no es algo superior en el interior, excepto cuando observamos el número de partos vaginales, destacando que en todos los casos se alcanzan los procedimientos mínimos suficientes para la aprobación de los semestres.

\section{Discusión}

En el proceso de la formación médica existen muchos factores determinantes de la calidad formativa. El pri- mero es el caudal de conocimientos básicos y la integración de los nuevos y diversos conocimientos que surgen del avance científico. Por otro lado, tener delimitado qué es lo que se debe enseñar, definiendo claramente las responsabilidades tanto del docente como del educando; la autonomía gradualmente ascendente que es necesario lograr en el proceso, y finalmente los escenarios de aprendizaje ${ }^{(8)}$. En la estrategia utilizada en el proceso de descentralización de la CGA, se objetivaron claramente los contenidos y la metodología para la adquisición del conocimiento de posgrado ${ }^{(5)}$, se promovió el compromiso docente con el logro de una red de docentes asociados y médicos colaboradores de la FM, y se formalizaron, con las diferentes instituciones, nuevos escenarios de aprendizaje que aseguraran el alcance de las competencias exigidas, generándose así ámbitos para lograr la formación de calidad.

Son múltiples los análisis sobre la importancia de la orientación educativa en el contexto de la actividad asistencial, y se afirma que la incorporación en la actividad académica se refleja en sus procesos y en los resulta$\operatorname{dos}^{(9)}$. Nosotros consideramos que la incorporación formal en la docencia de instituciones que clásicamente no han estado involucradas en la formación médica, promueve la innovación, la evaluación de resultados y la educación médica continua.

El desarrollo de un plan de descentralización en la formación de recursos humanos en ginecología desde la CGA a partir del año 2010 ha permitido cambiar la relación con respecto a la formación de posgrados, logrando modificar radicalmente la visión centralista en cuanto a la formación en Montevideo y específicamente en el 
CHPR (centro asistencial de referencia en la ginecotocología nacional). Se ha transformado una realidad, desde un punto de partida en el año 2010 donde la formación de posgrados de la CGA se encontraba $100 \%$ centralizada en Montevideo, al presente, donde los posgrados que se forman en el interior del país superan en número a los residentes formados en Montevideo. A lo largo de estos años de implementación, durante los cuales ya existe más de una generación de posgrados que han logrado su título de ginecotocólogos con formación en el interior del país, creemos que se ha demostrado a partir de las evaluaciones de los posgrados, pero sobre todo del logro del objetivo final que es el título de la especialidad, que la formación es posible en los diferentes CEDA, con dispositivos docentes innovadores.

Si bien no es un punto que hayamos evaluado, sabemos que los profesionales médicos entrenados en centros de alta complejidad tienen más experiencia en el cuidado de ciertos casos que en los más prevalentes y simples ${ }^{(10)}$. Seguramente de acuerdo con las características asistenciales diferentes del CHPR, centro de referencia nacional de gineco-obstetricia, este cambio en la distribución de los escenarios asistenciales de aprendizaje impacte sobre la integralidad del abordaje de la especialidad.

De los elementos comparativos objetivos, como son los procedimientos quirúrgicos realizados por los posgrados, podemos afirmar que tanto en el servicio del CHPR-FM como en los CEDA, los números de procedimientos básicos exigidos para graduarse fueron equiparables y superaron los mínimos necesarios exigidos por el programa.

Del análisis de la expansión en el número de posgrados de ginecología, una de las preocupaciones iniciales se centraba en poder mantener una adecuada capacidad formativa, sin resentir la formación en los posgrados que ya cursaban, ni en las nuevas generaciones. En este sentido queremos remarcar que del análisis formativo (evaluaciones) que se logra en los nuevos CEDA, es completamente suficiente y cumple con los requerimientos de la especialidad como están indicados en el programa de formación del posgrado de ginecología de la CGA e incluso, en algunos aspectos, supera la actividad desarrollada en el CHPR, por lo que este cambio en la distribución de los posgrados en forma descentralizada, lejos de afectar negativamente el nivel de formación de especialistas, lo ha potenciado.

Por un lado, creemos destacable la incorporación de centros asistenciales privados en las actividades de formación de posgrados, sin que esto se haya visto como una amenaza en la asistencia y, por otro lado, confirmar la plena capacidad formativa de los mismos.
El proceso de descentralización de los escenarios de enseñanza-aprendizaje ha permitido la ampliación de cupos de posgrados sin resentir la calidad de formación en aquellos centros donde la CGA ya venía formando a sus posgrados, como es el CHPR.

La investigación en educación médica contribuye a comprender los procesos de aprendizaje y poder así mejorar sus resultados. En los últimos tiempos los docentes y las autoridades universitarias están considerando la evidencia en educación médica como una herramienta en la toma de decisiones ${ }^{(11)}$. Sin embargo, una de las barreras actuales es que muchas veces se compara la investigación en educación médica con la investigación biomédica y muchos tratan de aplicar estas metodologías y no pueden llevarse a cabo o adaptar la pregunta ${ }^{(12)}$. No obstante, si bien nuestra presentación no corresponde a una investigación, sino a la presentación de la experiencia, sí creemos que aporta a nuestra comunidad educativa que necesariamente debe modificar, adaptar estrategias e innovar en la formación de recursos humanos.

\section{Conclusiones}

La CGA, comprometida con el SNIS mediante su plan de descentralización de los escenarios de enseñanza aprendizaje, permite la formación en de ginecotocólogos distribuidos mayoritariamente fuera del CHPR. Se ha logrado aumentar el cupo de posgrados, contribuir a la formación médica continua y facilitar y fortalecer la asistencia a nivel local, con la expectativa, además, de que esto favorezca la radicación de especialistas en el interior del país.

\section{Abstract}

The Gynecotocology Clinic A (GCA) of the School of the School of Medicine is committed to the goals set in the health reform in terms of the training of postgraduates, and thus has focused on the decentralization of the educational scenario.

This study presents the decentralization strategy in the training of postgraduate students in gynecotocology of the GCA between 2010 and 2018 .

It describes the tools that allow keeping in touch with the posgraduate students and tutors by means of technology that regularly aids grand rounds, tutorial activities, remote clinics, etc. Also, a description of the distribution of achievements by psotgraduates is provided, as well as the achievement of skills in the different Associated Teaching Centers (CEDAs in Spanish).

Upon the assessment of performance of the CEDAs network of CGA, as well as the educational achievements in human resources, a conclusion is drawn as to the feasibility of increasing the number of postgraduates 
trained in gynecotocology appealing to structured decentralization strategies for the learning scenario.

\section{Resumo}

A Clínica Ginecotocológica A (CGA) da Faculdade de Medicina (FMed-UDELAR) está comprometida com os objetivos da reforma sanitária no que diz respeito à formação dos pós-graduandos, trabalhando para isso na descentralização dos cenários de ensino-aprendizagem.

Nesta publicação apresenta-se a estratégia de descentralização da formação dos pós-graduandos de ginecologia e obstetrícia da CGA no período 2010-2018.

Descreve-se as ferramentas que permitem manter o contacto entre pós-graduandos e supervisores utilizando tecnologias da comunicação para realizar regularmente discussão de casos clínicos, atividades de supervisão e tele clínicas. Por outro lado, apresenta-se a distribuição geográfica dos pós-graduandos, bem como os resultados da obtenção de destrezas nos diferentes Centros docentes Associados (CEDA).

A avaliação do funcionamento da rede de CEDA da CGA e dos resultados da formação de recursos humanos, permite concluir que é factível aumentar as vagas para pós-graduandos de ginecologia e obstetrícia utilizando a descentralização estruturada dos cenários de aprendizagem.

\section{Bibliografía}

1. Universidad de la República del Uruguay. Misión de la Facultad de Medicina. Disponible en: http://www.fmed.edu.uy/ institucional/mision. [Consulta: 20 noviembre 2018].

2. Uruguay. Poder Legislativo. Ley $\mathrm{n}^{\circ} 18211$. Sistema Nacional Integrado de Salud (13 de diciembre de 2007). Disponible en: https://legislativo.parlamento.gub.uy/temporales/leytemp6103585.htm [Consulta: 20 diciembre 2018].
3. Sollazzo A, Berterretche R. El Sistema Nacional Integrado de Salud en Uruguay y los desafíos para la Atención Primaria. Ciênc saúde coletiva 2011; 16(6): 2829-40. doi:10.1590/S1413-81232011000600021.

4. Uruguay. IMPO. Ley $\mathrm{n}^{\circ}$ 19301. Régimen de residencias médicas hospitalarias. Disponible en: https://www impo.com.uy/bases/leyes/19301-2014. [Consulta: 12 setiembre de 2018].

5. Nozar F, Fiol V, Coppola F, Briozzo L. Programa de formación del postgrado de la Clínica Ginecotocológica A. Montevideo: Oficina del Libro-FEFMUR, 2011.

6. Universidad de la República del Uruguay. Facultad de Medicina. Proyecto ECHO Uruguay. Disponible en: https://drive.goole.com/file/d/ 0B4XzBGF1CkbKMEx3a0t1b3V2djg/view [Consulta: 27 junio 2018].

7. Albanese MA, Mitchell S. Problem-based learning: a review of the literature on its outcomes and implementation issues. Acad méd 1993; 68(1):52-81.

8. Campos A. Las cuatro ruedas del carro de la excelencia. Desafíos y limitaciones en la educación médica. Educ méd 2016; 17(3):88-93.

9. Millán Núñez-Cortés J, Civeira-Murillo F, Gutiérrez-Fuentes JA. El hospital universitario del siglo XXI. Educ méd 2011; 14: 83-9.

10. Byrne L, Holt K, Richter T, Miller R, Nasca T. Tracking residents through multiple residency programs: a different approach for measuring residents' rates of continuing graduate medical education in ACGME-accredited programs. J Grad Med Educ 2010; 2(4):616-23. doi:10.4300/JGME-D-10-00105.1.

11. Norman G. Research in medical education: three decades of progress. BMJ 2002; 324(7353):1560-2.

12. Alves de Lima A. Investigación en educación médica: un análisis sobre conceptos básicos. Rev Hosp Ital B.Aires 2007; 27(1):1-6.

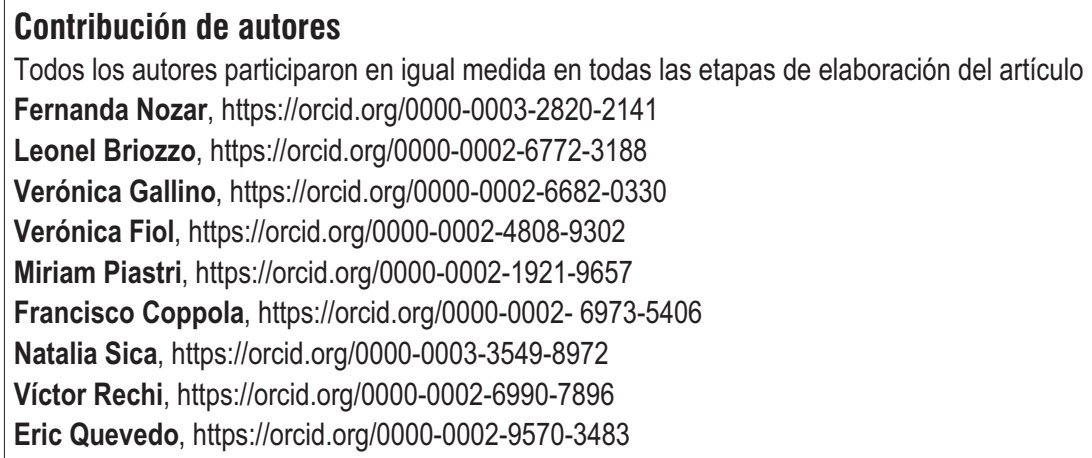

